\title{
Propuesta para el tratamiento de ecuaciones diferenciales de segundo orden aplicadas al sistema masa resorte. Una experiencia de aprendizaje mediada por GeoGebra durante la contingencia sanitaria en 2020
}

\section{Proposal for the Treatment of Second Order Differential Equations Applied to the Spring Mass System. A Learning Experience Mediated by Geogebra During the Health Contingency in 2020}

Maximiliano De Las Fuentes-Lara Universidad Autónoma de Baja California, México ORCID: http://orcid.org/0000-0002-1001-4663

Wendolyn E. Aguilar-Salinas Universidad Autónoma de Baja California, México ORCID: http://orcid.org/0000-0003-2223-9234

Received 07-17-20 Revised 08-01-20 Accepted 09-29-20 On line 09-30-20

*Correspondence

Email: maximilianofuentes@uabc.edu.mx
Cite as: 


\section{Resumen}

En este documento se presentan los resultados de un proyecto de investigación aplicado durante la pandemia del coronavirus en el año 2020 y el consecuente aislamiento social. El origen de la investigación versa por una parte en la imposibilidad de contar con la interrelación alumno docente de manera presencial en el sistema educativo universitario y por otra las dificultades cognitivas de los estudiantes de ingeniería para resolver aplicaciones de las ecuaciones diferenciales de segundo orden asociadas al fenómeno denominado sistema masa resorte. En este sentido, se diseñó e implementó una situación de aprendizaje que incorpora un programa interactivo elaborado con el software GeoGebra, con el propósito de mejorar los conocimientos y habilidades de los estudiantes en términos conceptuales alrededor de las aplicaciones de las ecuaciones diferenciales de segundo orden. Para evaluar el impacto de la puesta en escena de la situación de aprendizaje se diseñaron hojas de trabajo e instrumentos de medición diagnóstica y post-test, su implementación, revisión y análisis reveló que los alumnos logran asociar de forma adecuada el signo de la velocidad o pendiente de la recta tangente del contrapeso con su dirección y que el éxito de los estudiantes radica en trabajar con materiales que incluyan actividades que contengan un balance en las actividades cognitivas y el uso equitativo de los diferentes registros de representación, además de hacer énfasis en aspectos conceptuales del objeto matemático.

Palabras clave: GeoGebra; Sistema masa resorte; Ecuaciones diferenciales.

\section{Summary}

This paper presents the results of a research project implemented during the coronavirus pandemic in 2020 and the consequent social isolation. The origin of the research concerns, on the one hand, the impossibility of having the teaching student interrelationship in person in the university education system and on the other the cognitive difficulties of engineering students to solve applications of the second-order differential equations associated with the phenomenon called the mass-spring system. In this sense, a learning situation was designed and implemented that incorporates an interactive program developed with GeoGebra software, with the purpose of improving students' knowledge and skills in conceptual terms around the applications of second-order differential equations. To assess the impact of the learning situation staging, worksheets and instruments of diagnostic and post-test measurement were designed, their implementation, review and analysis revealed that students manage to adequately associate the sign of the speed or slope of the straight tangent of the counterweight with their direction and that the success of students lies in working with materials that include activities that contain a balance in cognitive activities and equitable use of different representation records, as well as emphasizing conceptual aspects of the mathematical object.

Keywords: Geogebra; Spring Mass System; Differential Equations.

\section{Introducción}

En la formación profesional del ingeniero la carga curricular incluye asignaturas del área de matemáticas: álgebra lineal, cálculo diferencial, cálculo integral, ecuaciones diferenciales (ED), entre otras. En el caso de las ED, el estudio y el aprendizaje de las mismas permiten modelar, comprender y avanzar en el conocimiento de diversos fenómenos de la naturaleza: crecimiento y decrecimiento poblacional, variación de temperatura de los cuerpos, propagación de virus, sistemas masa-resorte, iluminación, son ejemplos comunes de ello. Uno de los tópicos a estudiar 
durante el curso de ED es el sistema masa resorte (SMR) que tiene variadas implicaciones prácticas y profesionales, por ejemplo: los sistemas de amortiguamiento de vehículos de transporte cotidiano y de carga como autobuses y aviones, los análisis sísmicos en estructuras habitacionales, médicas o de negocios, la fabricación de productos por medio de robots o maquinaria pesada requiere tanto para su estabilidad como para su producción misma el análisis y diseño de piezas, uniones, brazos o soportes. Por tal motivo es de importancia su estudio teórico y comprensión por parte de los futuros ingenieros.

La unidad de aprendizaje de ED presenta conceptos que son difíciles de comprender por los estudiantes si no los pueden visualizar, como es el efecto de la variabilidad. Cistac, Bongianino, Filippi y Kovac (2009) indican que precisamente en la etapa de construcción inicial de las capacidades tecnológicas es esencial enlazar los conceptos matemáticos con el proceso físico lo que conlleva al cambio en las representaciones semióticas. La utilización de la tecnología en la enseñanza permite manipular dinámicamente los objetos matemáticos $(\mathrm{OM})$ en diferentes contextos de representación, de hecho la capacidad de modelado matemático de un estudiante depende del manejo eficiente de los distintos registros de representación (Báez, Blanco \& Pérez, 2015b). Investigadores en el área de las matemáticas para ingeniería identificaron que los conocimientos previos al cálculo de los estudiantes es más procedimental que conceptual, además, los resultados de sus estudios evidencian que el proceso de enseñanza y aprendizaje del cálculo en las aulas así como la propia evaluación es predominantemente procesal (Areaya \& Sidelil, 2012). También se encontró que los estudiantes de matemáticas privilegian el enfoque procedimental simbólico ya que se les dificulta interpretar las gráficas de las funciones y sus derivadas, toda vez que el vínculo conceptual no se prioriza (Habre \& Abboud, 2006; Hashemi, Abu, Kashefi \& Rahimi, 2014; Báez, Blanco \& Pérez, 2015a), el desequilibrio entre el conocimiento operacional y el conceptual es un factor trascendente que contribuye a la debilidad del estudiante para obtener información sobre la variación (Abbey, 2008). Una investigación llevada a cabo por Zerrin y Sebnem (2010) en la que se diseñó e implementó una situación de aprendizaje (SA) para el concepto de función utilizando una aplicación en el Software GeoGebra (SG), mostró que los estudiantes del grupo experimental fueron más exitosos en cuanto a sus logros académicos debido a que la visualización y la dinámica de las formas geométricas que permite la aplicación diseñada en el SG aumenta significativamente la atención de los alumnos. El SG permite diseñar diferentes aplicaciones interactivas que se pueden usar como herramientas en la enseñanza de la matemática (Caligaris, Schivo \& Romiti, 2015) y experimentar con modelos significativos utilizando múltiples representaciones y herramientas de modelado (Hashemi et al., 2014) y que mejoran las habilidades de razonamiento y la visualización de los estudiantes (Bhagat \& Chang, 2015).

La aplicación del SG permite a los estudiantes comprender los conceptos geométricos, propiciando el intercambio de experiencias y reflexiones que enriquezcan y mejoren la calidad del aprendizaje de las matemáticas (Torres \& Racedo, 2014; Ponce, Matthews \& Adams, 2018), presenta un gran potencial para el tratamiento de la interpretación, ya que permite discriminar la congruencia entre las características visuales y la semántica de la expresión algebraica (GómezBlancarte, Guirette \& Morales-Colorado, 2017). El SG es una herramienta que permite a los estudiantes tener un aprendizaje interactivo y autónomo (Arango, Gaviria \& Valencia, 2015). El uso del SG en la enseñanza de las matemáticas en los diferentes niveles educativos es un factor importante para promover el aprendizaje permanente (Zerrin \& Sebnem, 2010; Zengin, Furkan \& Kutluca, 2012). 
Investigaciones en el uso del SG concluyen que se puede utilizar como una herramienta eficaz en el aprendizaje a través de la visualización, promueve el aprendizaje y mejora la comprensión, los investigadores consideran que se trata de una forma de utilizar la tecnología con ayudas visuales y propicia en los estudiantes la interacción con los conceptos matemáticos (Saha, Ayub \& Tarmizi, 2010; Córdoba, Castrillón \& Rojas, 2015; Chen \& Wu, 2020). Sin lugar a duda la versatilidad del SG sugiere su utilización para mejorar el conocimiento y las habilidades de los estudiantes en torno al planteamiento y resolución de enunciados de problemas en el campo de las ED.

En el mes de marzo de 2020 se declara la pandemia del coronavirus en México, a partir de esa fecha las autoridades sanitarias y educativas en México y específicamente en Baja California indicaron suspensión de clases presenciales y sugirieron permanecer en aislamiento social, a laborar y dar continuidad al ciclo escolar vigente mediante diversas plataformas. Las autoridades de la Universidad Autónoma de Baja California (UABC) giraron instrucciones para trabajar con la plataforma BlackBoard (BB), ello implicó la planeación y diseño puntual de diversas SA y diversos mecanismos de evaluación para atender académicamente a los estudiantes.

Además del problema de la contingencia sanitaria los resultados que se obtienen en los cursos de matemáticas de prácticamente cualquier nivel escolar no son satisfactorios, para los estudiantes mexicanos los resultados que se obtienen en las evaluaciones están por debajo del promedio internacional (PISA, 2012, 2015), aunque existen muchos factores que intervienen en tales desempeños se han detectado deficiencias para representar y efectuar tratamientos y conversiones entre los diferentes registros de representación de los conceptos matemáticos, lo cual parece deberse a la preponderancia de la enseñanza de las matemáticas basadas en un enfoque tradicional.

A partir de los antecedentes formulados y con énfasis en no contar con la interrelación presencial entre el docente y el alumno, se plantea el diseño e implementación de una SA que incluye hojas de trabajo y un programa interactivo desarrollado en el SG que permita al estudiante universitario de ingeniería en su curso de ED promover de forma dinámica la vinculación de los diferentes registros de representación (gráfica, numérica, algebraica y física virtual) y las actividades cognitivas (AC) en torno al fenómeno SMR y que implican la resolución de ED lineales de segundo orden. En razón de lo anterior el objetivo de esta investigación es evaluar el impacto que se logra en los estudiantes sobre las AC a partir de la utilización de la SA propuesta. Por tanto, se pretende aportar una propuesta pedagógica que incluye el uso del SG como mediador entre los estudiantes y conceptos matemáticos asociados a la resolución de problemas sobre el fenómeno SMR y las ED lineales de segundo orden.

De manera colateral se tienen los siguientes objetivos particulares:

- Determinar qué AC mejora sustancialmente en el estudiante a partir del programa interactivo desarrollado con el SG.

- Identificar el registro de representación original con mayor dificultad para el estudiante a partir de la aplicación interactiva desarrollada en el SG.

- Determinar elementos predictivos sobre el éxito de los estudiantes en sus evaluaciones en torno al fenómeno SMR y las ED de segundo orden. 


\section{Marco teórico}

El diseño de la SA se basa en la Teoría de Representaciones Semióticas (TRS) de Duval (2000, 2006a, 2006b) y los avances logrados en el campo tecnológico específicamente con el SG y su aplicación en la enseñanza y el aprendizaje de las matemáticas (Zerrin \& Sebnem, 2010; Zengin et al., 2012; Córdoba et al., 2015; Nazihatulhasanah \& Nurbiha, 2015; Caligaris et al., 2015). La SA diseñada y utilizada se considera una estrategia instruccional de acuerdo a Feo (2010) toda vez que la interrelación presencial entre el docente y estudiante no es indispensable y se apoyan en un recurso instruccional tecnológico.

Desde la perspectiva de la TRS, los OM no son directamente accesibles a la percepción, consecuentemente para su estudio y tratamiento se requiere contar representaciones de los mismos, las representaciones externas a las que hacemos alusión pueden ser de carácter geométrico, algebraico y numérico. Como lo señala el mismo Duval (2000), el uso de sistemas de representaciones semióticas para el pensamiento matemático es esencial, debido a que a diferencia de otros campos de conocimiento (biología, geología, física), no existen otras maneras de lograr el acceso a los OM sino a través de representaciones. Estos procesos de representación, tratamiento y conversión permiten exteriorizar las representaciones mentales de los individuos, motivando la retroalimentación y mejoramiento de las mismas, que conduce a la formación de preceptos matemáticos y a la conceptualización del OM. La AC de representación constituye una marca o conjunto de marcas perceptibles e identificables respecto de un OM, el tratamiento es la transformación de la representación dentro del mismo registro de representación, y la conversión es la transformación de la representación en otra representación de un registro diferente al original pero que conserva su esencia (Duval, 2006a).

Otra parte importante de las SA es estar orientados a la comprensión de contenidos conceptuales y procedimentales (Feo, 2010). Al respecto Contreras y Backhoff (2004) mencionan que la parte conceptual se refiere a las características, atributos ontológicos, la definición del propio concepto y la apropiación de una regularidad. Para Sfard (1991) la visión estructural o conceptual se caracteriza por un ente que posee rasgos propios y es integradora, y la visión operacional o procedimental se matiza por acciones y algoritmos, que es dinámica y secuencial. Contreras y Backhoff (2004) la refieren como al dominio de las fases que implica la secuencia de operaciones involucradas y la utilización de algoritmos.

\section{Metodología}

A partir de los referentes teóricos descritos se asumen los siguientes supuestos como base para el diseño y puesta en escena de la SA que se describe en los párrafos siguientes. El primero es que el éxito de un estudiante en el curso de ED particularmente en el tratamiento del SMR se debe al dominio conceptual sobre los $\mathrm{OM}$ y la AC de conversión. El segundo se refiere al involucramiento de los recursos tecnológicos como mediador entre el sujeto y el objeto de conocimiento.

Además de integrar información a partir de la experiencia y práctica docente del equipo de trabajo en el área de las ED, se consideró la aplicación de un instrumento de medición diagnóstico (IMD), se revisó el contenido de fuentes bibliográficas respecto a los artículos, memorias y documentos existentes sobre los efectos de algunas tecnologías y software de matemáticas (exclusivamente sobre el $\mathrm{SG}$ ) que han trascendido en torno a aspectos 
motivacionales y los conocimientos que logran los estudiantes en diferentes tópicos de matemáticas.

Dado que en el estudio de las matemáticas para ingeniería se recurre de forma importante a distintas representaciones (numérica, gráfica, algebraica) y se considera que la aprehensión conceptual está vinculada al dominio de las AC de representación, tratamiento y conversión, se optó por fundamentar el diseño de la SA en la TRS de Duval (2000, 2006a, 2006b). Desde esta perspectiva el programa interactivo en GeoGebra y la SA están alineados a tales referentes teóricos, el programa interactivo y la SA buscan promover la coordinación de registros de representación, así como también la exploración, reflexión y la aprehensión conceptual.

\section{Información a partir de la experiencia docente.}

Durante los inicios del semestre 2020-1 los investigadores solicitaron a tres profesores que imparten la unidad de aprendizaje de ED en la Facultad de Ingeniería de Mexicali (FIM) de la UABC y con experiencia docente mayor a 5 años la tarea particular de describir todas aquellas dificultades que han observado en los estudiantes de ingeniería en torno al SMR y la resolución de ED de segundo orden asociadas.

\section{Instrumento de medición diagnóstico.}

El diagnóstico es esencial para la planeación eficaz de la instrucción en general y para el diseño de la SA en particular, así como para evidenciar los cambios producto del aprendizaje adquirido por los estudiantes. Con el propósito de establecer las condiciones de los estudiantes en cuanto a sus conocimientos previos al inicio del tratamiento del fenómeno SMR y establecer de manera paralela las habilidades en cuanto a su AC para la representación, tratamiento y conversión de objetos matemáticos, se diseñó y aplicó un IMD con la consideración de los criterios de Contreras y Bachhoff (2004) para la generación de reactivos en la construcción de exámenes del tipo criterial alineado con el currículum, que involucra contenidos conceptuales y procedimentales, los cuales han sido estudiados y tratados por los estudiantes en cursos de cálculo diferencial, integral y ED.

\section{Situación de aprendizaje.}

La SA diseñada se basa en Feo (2010) y consta de cuatro momentos: inicio, desarrollo, cierre y evaluación. En el momento de inicio se contactó a los estudiantes del grupo de ED mediante la plataforma BB, con la cual se les comunicó que se daría tratamiento al estudio del SMR y que los materiales (hojas de trabajo) estarían disponibles para descarga. Dentro de los materiales, se les proporcionó la liga de la página web del programa interactivo en GeoGebra como apoyo al estudio y tratamiento del SMR, las instrucciones de uso y fechas de entrega de los reportes correspondientes. El segundo momento tuvo una duración de dos semanas, en las cuales los estudiantes podían utilizar los materiales en el espacio de tiempo que consideraran conveniente y colaborar con sus compañeros a través de los diversos medios de comunicación (Facebook, Whatsapp, correo electrónico, etc.). También se solicitó a los estudiantes como actividad de reforzamiento del estudio del SMR y uso de las ED la resolución y entrega de un problemario que incluyó 10 enunciados de problemas, todos alusivos al SMR. El momento de cierre corresponde a la entrega tanto del problemario como de las hojas trabajo de todo el grupo de 
estudiantes, la entrega implica resolver los 10 problemas, responder las hojas de trabajo, digitalizar y subir a la plataforma BB. La evaluación como parte del cuarto momento le corresponde al profesor y en este caso implicó la revisión de los problemas y de las respuestas de todas las hojas de trabajo, así como también la aplicación de un instrumento de medición post-test (IMP), dicho instrumento se subió a la plataforma BB y se comunicó a los estudiantes el día, hora, duración y requerimientos para su contestación.

\section{Diseño del programa interactivo mediante el software GeoGebra.}

El programa interactivo diseñado con el SG es un SMR que puede describir el desplazamiento de un contrapeso a partir del establecimiento de los parámetros de masa, amortiguamiento, rigidez y condiciones iniciales de posición y velocidad.

El programa interactivo con el SG consta de dos vistas (figura 1), una vista gráfica1 y una grafica2, la vista gráfica1 contiene un sistema de coordenadas bidimensional $(t, x(t))$ que describe la posición del contrapeso en términos del tiempo transcurrido, está asociado un SMR cuyo desplazamiento corresponde a la construcción dinámica de la gráfica, de manera adicional cuenta con la manipulación de una recta tangente asociada tanto a la velocidad del contrapeso como a la dirección del mismo. La vista gráfica2 consta de cuatro partes: la primera incluye cinco cuadros de edición de datos, posición inicial, velocidad inicial, masa, factor de amortiguamiento y rigidez; la segunda parte se refiere al control de la simulación, a partir de un tiempo cero, pausa y el inicio o continuación de la simulación; la tercera parte incluye cálculos que realiza el programa interactivo (posición y velocidad del contrapeso) a partir de la variable independiente tiempo y los datos de masa, rigidez y amortiguamiento; finalmente, la cuarta parte permite incorporar una recta tangente a la simulación que está asociada a la velocidad del contrapeso.

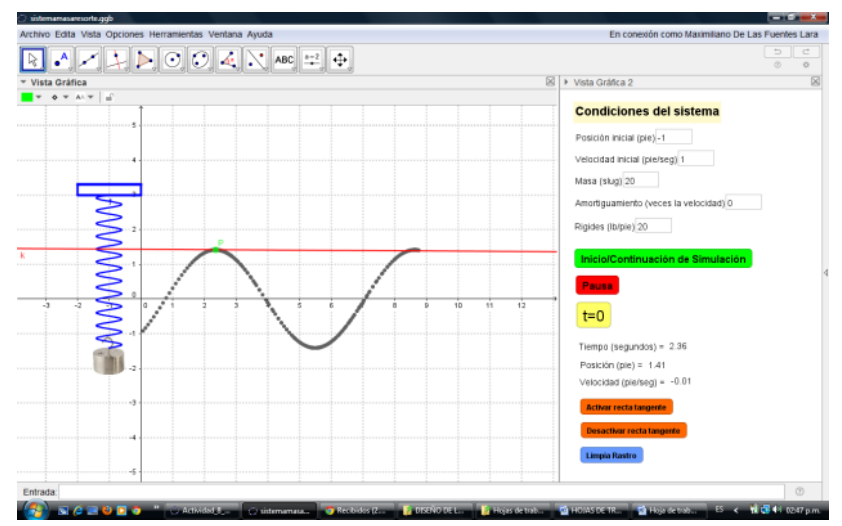

Figura 1. Programa interactivo en GeoGebra

Los contenidos matemáticos involucrados en el diseño de la SA son los siguientes: ED ordinarias lineales de segundo orden homogéneas, rigidez, amortiguamiento, fuerza, desplazamiento, masa, función, función derivada, ecuación de movimiento, condiciones iniciales, periodo, amplitud, frecuencia, velocidad y aceleración.

\section{Hojas de trabajo.}

La SA consta de 4 hojas de trabajo, cada una corresponde a un tipo de movimiento, a saber: no amortiguado, sobreamortiguado, críticamente amortiguado y subamortiguado, hojas de trabajo 
1, 2, 3 y 4 respectivamente. Los objetivos de aprendizaje de las 4 hojas de trabajo son los siguientes:

- Determinar e interpretar las condiciones iniciales de posición y velocidad.

- Determinar la ED que modela el sistema (ED y condiciones iniciales).

- Determinar la solución general y particular de la ED.

- Asociar la gráfica de la ecuación de movimiento con el movimiento vertical del contrapeso.

- Asociar la posición del contrapeso con la gráfica.

- Asociar los máximos y mínimos de la gráfica con relación a la posición del contrapeso y condición de contracción y estiramiento del resorte.

- Asociar el signo de la pendiente de la recta tangente con la dirección del contrapeso.

- Asociar el valor de cero de la pendiente de la recta tangente con la posición máxima o mínima del contrapeso.

- Calcular la posición del contrapeso en un momento determinado.

- Calcular la velocidad y dirección del contrapeso en un momento determinado.

- Identificar el tipo de movimiento del SMR.

- Calcular el periodo, frecuencia y amplitud del SMR.

- Calcular el momento del desplazamiento extremo del contrapeso y su posición.

- Explicar el efecto de la variación de los elementos de la ED.

\section{Sujetos.}

La SA se puso en escena durante el semestre 2020-1 (febrero-junio 2020) con 34 estudiantes del tercer semestre de la FIM en la UABC en la asignatura de ED, en dicha asignatura se trata el SMR como una aplicación de las ED de segundo orden. Antes de llevar a cabo la experimentación con la SA diseñada los estudiantes ya habían trabajado con problemas de aplicación relacionados con temperatura, crecimiento y decrecimiento, mezclas y datación, problemas que se modelan con ED de primer orden. También se solicita a los estudiantes como actividad previa la lectura del SMR y sus respectivos movimientos amortiguado y no amortiguado, material y bibliografía que se les proporcionó con la debida antelación.

\section{Instrumento de medición post-test.}

El IMP está compuesto por 18 reactivos de opción múltiple, en el que se pide al estudiante elegir de entre 4 respuestas la que es correcta. Cada reactivo en el instrumento es independiente, toda vez que contiene la información necesaria para plantearlo y responderlo. El IMP es criterial, ya que tiene el propósito de evaluar el aprendizaje, informando sobre qué puede hacer o no el examinado. El IMP se diseñó bajo las mismas consideraciones que el IMD, a diferencia que se aboca a determinar los logros alcanzados por los estudiantes en cuanto a las AC de representación, tratamiento y conversión en torno a los conceptos matemáticos del SMR, también se clasificó cada reactivo como conceptual o procedimental con el propósito de indagar sobre qué tipo de reactivos les cuesta mayor dificultad a los estudiantes y si es posible predecir su éxito a partir del tipo de reactivo. Se realizó un análisis de confiabilidad mediante el coeficiente de Kuder-Richarson (KR-20), el cual permite obtener la confiabilidad de un instrumento a partir de los datos obtenidos en una sola aplicación (Corral, 2009). De manera adicional se calculó el coeficiente delta de Ferguson que mide el poder de discriminación de una prueba completa. El índice de dificultad (ID) está relacionado con la proporción de estudiantes que resuelven correctamente un reactivo, y se calcula de acuerdo a Crocker y Algina (1986). El 
índice de discriminación (IDC) del reactivo permite discriminar entre aquellos estudiantes que obtuvieron altas calificaciones en la prueba y aquellos que obtuvieron bajo puntaje, está relacionado con la posibilidad de responder correctamente el reactivo aquellos estudiantes con un desempeño en general sobresaliente en la prueba, situación opuesta para el caso de los estudiantes con un desempeño deficiente. También se calculó el coeficiente de correlación del punto biserial (rpbis), para algunos investigadores este coeficiente es un indicador de validez predictiva, en donde se relaciona la respuesta a un reactivo por un estudiante y el resultado que obtuvo de la prueba (Henrysson, 1971; Molina, Wizner, Lacave \& Gallardo, 2015), este indicador psicométrico se calcula de acuerdo al modelo de Backhoff, Larrazolo y Rosas (2000). La validez de contenido tanto del IMD como del IMP se garantizó con la participación de jueces expertos (profesores de cálculo y de ED con experiencia docente mínima de 5 años) y método de consenso grupal (Corral, 2009).

\section{Análisis de resultados y discusión}

En este apartado se ofrecen los resultados de la consulta con los profesores de mayor experiencia con estudiantes en los cursos de ED, los resultados de la aplicación del IMD, un análisis cualitativo de los resultados de los estudiantes en las hojas de trabajo y se documentan los resultados que obtuvieron los estudiantes en el IMP. Como producto de esta investigación se espera determinar el impacto en los conocimientos y AC de representación, tratamiento y conversión de los alumnos a partir del uso de un programa interactivo desarrollado con el SG para abordar ED lineales homogéneas de segundo orden en la resolución de problemas asociados al SMR. Así como también, la obtención de un mayor conocimiento tanto de la problemática que se presenta cuando se brinda autonomía total al aprendizaje del estudiante y se incorpora una SA que incorpora tecnología en el proceso de enseñanza.

\section{Resultados de la experiencia docente.}

A partir de la experiencia y práctica docente del equipo de trabajo en el área de las ED, se ha observado a priori en los estudiantes las siguientes dificultades: aprehensión conceptual limitada de los objetos matemáticos debida en parte al énfasis en la praxis algorítmica e insuficiente trabajo de conversión de registros de representación durante las clases de esta asignatura, la AC de conversión evidencia la mayor dificultad para los estudiantes cuando esta se presenta como necesaria para la resolución de un problema, la AC de representación se posiciona como la de menor dificultad y secundariamente la AC de tratamiento.

Los problemas de aplicación de ED son los que causan mayor dificultad a los estudiantes, particularmente cuando requieren modelar el fenómeno SMR, es decir, traducir del lenguaje natural a un lenguaje matemático. En un SMR el estudiante tiene dificultades para identificar la velocidad y la aceleración del contrapeso como la primera y segunda derivada de la función posición, así como asociar la dirección del contrapeso con el signo de la velocidad y vincular la forma de la ED con el tipo de movimiento.

\section{Calidad del instrumento de medición diagnóstico y resultados de la aplicación.}

El IMD consta de 32 reactivos de opción múltiple y su contenido versa sobre tópicos esenciales de cálculo diferencial, integral y ED. El número de estudiantes que participaron en el diagnóstico fue de 33. Para el cálculo de la confiabilidad se utilizó el método de KR-20, el cual resultó $r=0.80$, para Ding, Chabay, Sherwood y Beichner (2006) es satisfactorio cuando 
$r \geq 0.80$. La distribución de los puntajes totales se calculó mediante la prueba delta de Ferguson, se obtuvo 0.96, lo que satisface el criterio establecido (Ding et al., 2006; Engelhardt, 2009). El promedio del ID es de $0.70 \pm 0.16$ (media \pm desviación estándar), resulta un nivel medio de dificultad y adecuado (Backhoff, Larrazolo y Rosas, 2000; Ding y cols., 2006; Engelhardt, 2009). El promedio del IDC es $0.37 \pm 0.14$ (media \pm desviación estándar), el cual cae dentro de una calificación considerada como excelente (Backhoff et al., 2000; Ding et al., 2006). A partir de los resultados de la aplicación de este IMD se encontró que los estudiantes tienen mayormente dificultad para traducir al lenguaje natural una ED de primer orden, identificar el concepto de derivada, determinar el tipo de solución que produce una ecuación de segundo grado, identificar la condición de linealidad en una ED y obtener la solución general de una ED lineal homogénea de segundo orden.

\section{Análisis cualitativo de las respuestas de los estudiantes en las hojas de trabajo.}

A partir de la revisión de las respuestas que los estudiantes plasmaron en sus hojas de trabajo, se considera pertinente incluir algunas de ellas aquí como evidencia particular, también se describirá de manera general los aciertos y deficiencias en torno a los objetivos de aprendizaje asociados al SMR.

En la hoja de trabajo 1 se les cuestiona a los estudiante sobre la interpretación de los máximos y mínimos de la gráfica con relación a la posición del contrapeso, algunas de las respuestas se exhiben en las figuras 2,3 y 4.

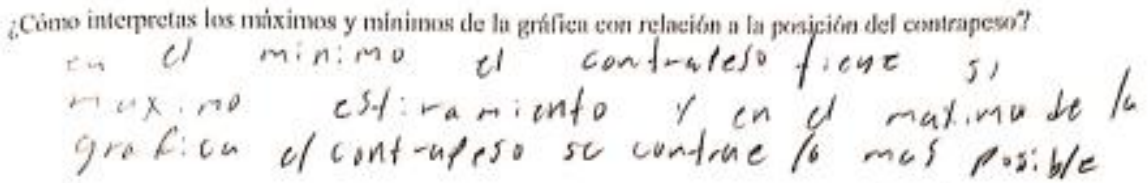

Figura 2. Respuesta del alumno A

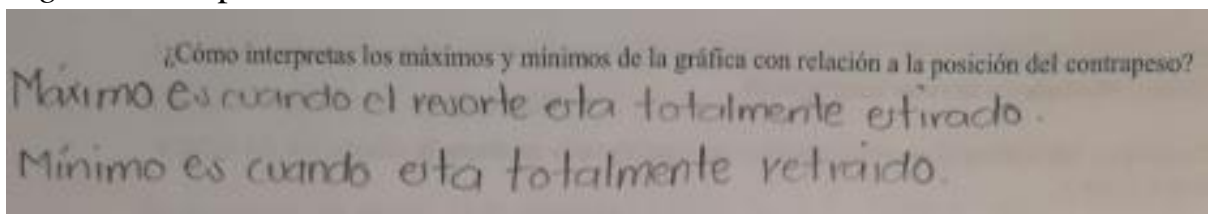

Figura 3. Respuesta del alumno C

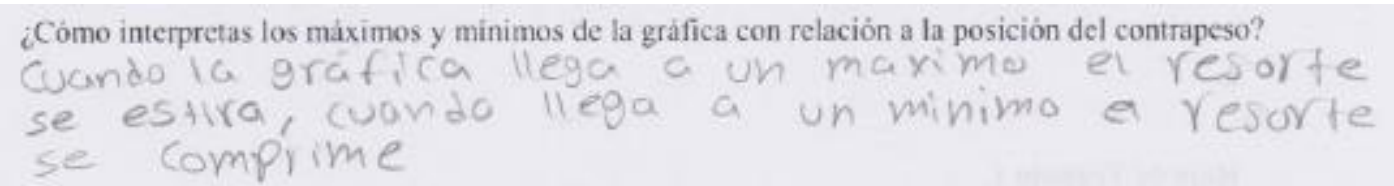

Figura 4. Respuesta del alumno N

Los alumnos $\mathrm{C}$ y $\mathrm{N}$ asocian correctamente el comportamiento gráfico que se produce con el movimiento libre no amortiguado del contrapeso asociado a la condición de contracción o estiramiento del resorte, el alumno A no ha logrado hacer la asociación correcta.

Al continuar con el desarrollo el alumno A suprime parcialmente su error con la respuesta a la siguiente pregunta: ¿En qué posición de la gráfica debes colocar el punto para que el resorte este totalmente contraído? (Figura 5). 
¿Lin qué posición de la gráfica dehes colecar el punto para que el resorte este tolalmente exntraído?

$$
\text { in Cris|quiera de sus min.mos }
$$

Figura 5. Respuesta del alumno A

Las respuestas de los alumnos $\mathrm{C}$ y $\mathrm{N}$ son consistentes (Figura 6 y 7), el programa interactivo en el SG permite que el estudiante modifique la condición del resorte (totalmente estirado o totalmente contraído) mediante la manipulación de la recta tangente de manera que pueda asociarlo con un mínimo o un máximo en la gráfica.

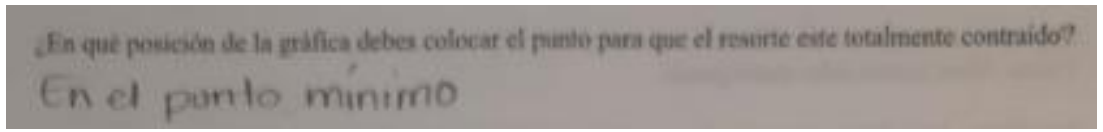

Figura 6. Respuesta del alumno C

¿En qué posición de la gráfica debes colocar el punto para que el resorte este totalmente contraido? en los minimos

Figura 7. Respuesta del alumno N

La mayoría de los estudiantes logró esta asociación y lo evidenció en sus hojas de trabajo. La visualización que se logra a partir de la tecnología y la aplicación interactiva juega un papel preponderante, Fatimah, Norazzila y Rohani (2013) señalan que los estudiantes superan dificultades cuando se integra tecnología en la enseñanza y el aprendizaje se centra en el estudiante.

En la hoja de trabajo 1 se tiene el siguiente cuestionamiento: en un tiempo de 6 segundos ¿Cuál es el valor de la velocidad? y ¿Hacia dónde se dirige el contrapeso?. En general la lectura tomada por los estudiantes sobre la velocidad es correcta, sin embargo la dirección del contrapeso no lo fue, solamente el $40 \%$ acertó en declarar la dirección del contrapeso. Estos estudiantes que lo hicieron correctamente, observaron el movimiento vertical hacia abajo del contrapeso y lo vincularon con el signo positivo de la primera derivada (Figura 8, 9 y 10).

En $\mathrm{t}=6$ segundos. ¿Cuál es el valoe de la velocidad?, ¿Hacia dónde se dirige el contrapeso?

$$
0.68 \% \mathrm{f} / \mathrm{s} \text { haciu abajo }
$$

Figura 8. Respuesta del alumno A

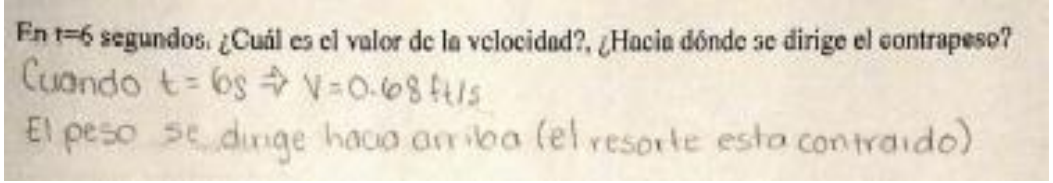

Figura 9. Respuesta del alumno B

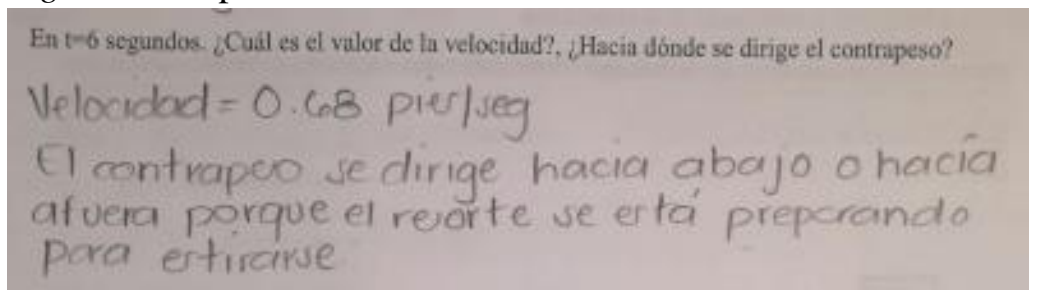

Figura 10. Respuesta del alumno C

En la hoja de trabajo 3 se tiene el siguiente enunciado de problema: un resorte con rigidez de 0.25 libra por pie se le sujeta un contrapeso de 32 libras fuerza, el medio a través del 
cual se mueve ofrece una resistencia numéricamente igual a una vez su velocidad instantánea. La pesa se suelta de un pie por arriba de la posición de equilibrio con una velocidad de 2.5 pie por segundo hacia abajo. Se solicita a los estudiantes que extraigan la información del problema respecto a la masa, rigidez, factor de amortiguamiento, posición inicial y velocidad inicial. Las respuestas de los alumnos se observan en las figuras 11 y 12.

Extrac la intormación correspondiente de: Masa, rigidez, amortiguamiento, posición inicial y velocidad inicial.

$\begin{array}{ll}\text { Masa: I sfoy Rigidez: } 0.25 / 6 & \text { Amortiguamiento: } 1 \\ \text { Posición inicial: }-1 & \text { Velocidad inicial: } 2.5 \\ \text { Figura 11. Respuesta del alumno A } & \end{array}$

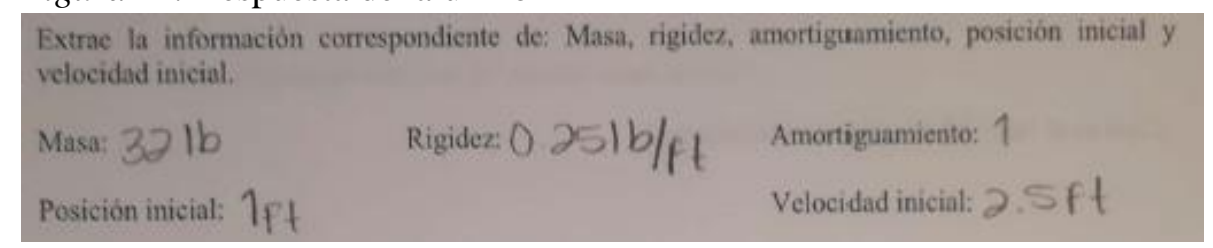

Figura 12. Respuesta del alumno C

La respuesta del alumno A es totalmente correcta sin embargo el alumno C no hizo la conversión de la masa de libras fuerza a slug y la posición inicial la consideró positiva por estar sobre la posición de equilibrio.

En la misma hoja de trabajo 3 se solicitó la solución particular de la ED, en las respuestas de los alumnos $\mathrm{B}$ y $\mathrm{N}$ se evidencia el modelado de forma adecuada toda vez que parten con la solución general de la ED y las condiciones iniciales correctas (Figura 13 y 14), según Guerrero-Ortíz, Mejía-Velasco y Camacho-Machín (2016) el uso de conexiones entre las diferentes representaciones matemáticas con apoyo del SG es un medio adecuado para establecer la relación entre el modelo y el contexto. Los errores que no permitieron a los alumnos B y $\mathrm{N}$ obtener la solución particular de la ED son de corte procedimental.

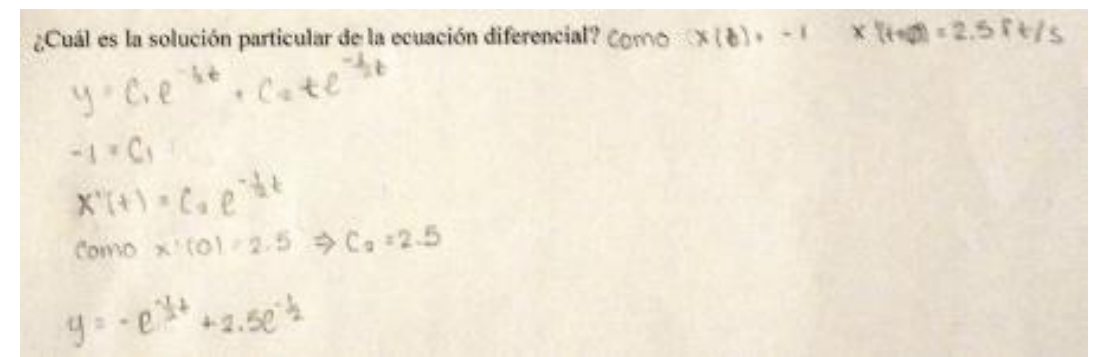

Figura 13. Respuesta del alumno B

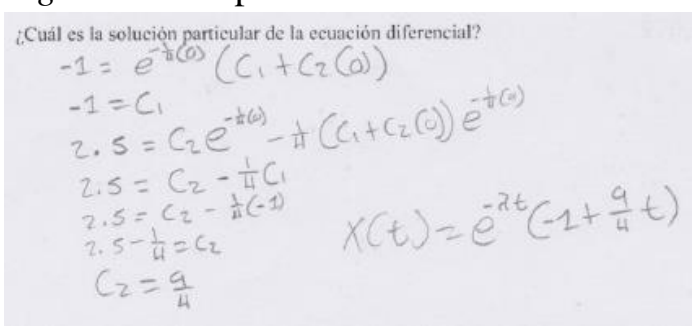

Figura 14. Respuesta del alumno N 
En la hoja de trabajo 4 se solicitó a los estudiantes la activación de la recta tangente y recorrer la curva para identificar en el intervalo de tiempo de dos a cinco segundos, sí la gráfica de la función es creciente o decreciente y establecer la dirección del contrapeso. Las respuestas indican que se tiene conflicto con la asociación de función creciente, velocidad positiva del contrapeso, dirección del contrapeso y condición de contracción o estiramiento del resorte (Figura 15 y 16).

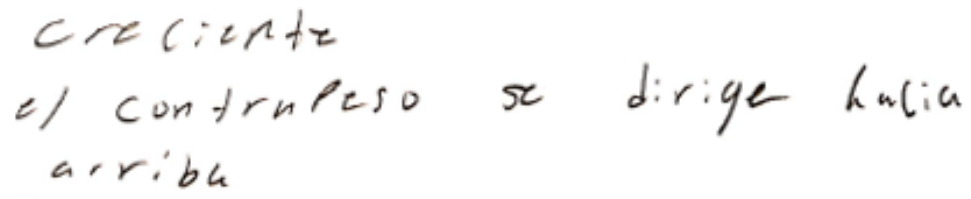

Figura 15. Respuesta alumno A

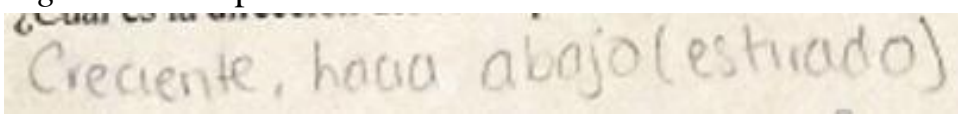

Figura 16. Respuesta alumno B

Además de las deficiencias y aciertos presentados de manera particular con algunos estudiantes, de manera general se documentan las siguientes observaciones sobre el desempeño de los estudiantes en las hojas de trabajo.

- La mayoría de los estudiantes extrajeron correctamente los elementos de masa y rigidez que definen la ED y que modela el SMR con movimiento libre no amortiguado, no así para el caso en el que el SMR cuenta con amortiguamiento.

- Los estudiantes asociaron de manera correcta el movimiento vertical del contrapeso con el comportamiento oscilatorio de la gráfica.

- Los estudiantes apreciaron que en el punto máximo o en el punto mínimo la pendiente de la recta tangente o la velocidad del contrapeso es de 0 , además de identificar correctamente la lectura del tiempo en esa condición.

- En el punto máximo, los estudiantes observaron la condición del resorte y asociaron que cuando en la gráfica se tiene un máximo el resorte se encuentra estirado a su límite, caso opuesto para el mínimo en donde el resorte se encuentra totalmente contraído.

- Se logró parcialmente que los alumnos asociaran adecuadamente el signo de la velocidad o pendiente de la recta tangente del contrapeso con su dirección.

- Algunos estudiantes no describieron la manera en que se calcula el desplazamiento extremo en virtud de que no asociaron directamente el término de desplazamiento extremo con un punto crítico.

- Los estudiantes lograron asociar el número de cortes de la gráfica con respecto al eje horizontal con el número de veces que el contrapeso pasa por la condición de equilibrio.

- Todos los estudiantes asociaron el comportamiento de la gráfica con la estabilización del sistema y la posición del contrapeso en el eje de referencia.

- Se encontró latente la dificultad de los estudiantes para transitar del lenguaje natural al lenguaje algebraico, algunos de los estudiantes no lograron determinar la ED y sus condiciones iniciales de posición y de velocidad.

- Se detectaron errores de manipulación algebraica que aún son muy comunes que los estudiantes cometan en el curso de ED. 
Para Hamidreza, Zaleha y Yudariah (2012) la visualización es la mayor dificultad que enfrentan los estudiantes que estudian matemáticas para ingeniería. El programa interactivo posibilita visualizar los OM que no son observados con tecnología de lápiz y papel.

Al comparar estos resultados con los objetivos de aprendizaje se puede pensar que el balance no es favorable, sin embargo hay que recordar que estos resultados son producto de una SA puesta en escena en un periodo de pandemia, con comunicación asíncrona y sin la presencia física del profesor para resolver e aclarar conceptos y procedimientos asociados al tema de estudio del SMR.

\section{Análisis del post-test.}

La confiabilidad del IMP se calculó mediante KR-20, resultó $r=0.86$, para Contreras y Backhoff (2004) y Ding y colaboradores (2006) es considerado como apropiado (mayor o igual que 0.85) en el caso de instrumentos estandarizados cuando se utiliza el método KR-20. La distribución de los puntajes totales se calculó mediante la prueba delta de Ferguson, se obtuvo un coeficiente de 0.95 lo que satisface ampliamente el criterio establecido (Ding et al., 2006; Engelhardt, 2009). El promedio del ID es de $0.63 \pm 0.17$ (media \pm desviación estándar), el cual resulta en un nivel medio de dificultad y adecuado (Backhoff et al., 2000; Ding et al., 2006; Engelhardt, 2009). El promedio del IDC es $0.53 \pm 0.17$ (media \pm desviación estándar), el cual cae dentro de una calificación considerada como excelente (Backhoff et al., 2000; Ding et al., 2006). Se cuenta con que el 78\% (14) de los reactivos tienen discriminación excelente, $5.5 \%$ (1) discriminación buena, $11 \%$ (2) cuenta con discriminación regular, mientras que el $5.5 \%$ (1) tiene discriminación mala.

El promedio de los coeficientes de correlación biserial de la prueba es $0.53 \pm 0.17$ (media \pm desviación estándar), el rpbis cumple de manera sobresaliente con la recomendación de los especialistas (Ding et al., 2006; Engelhardt, 2009) en cuanto a que el promedio del rpbis sea superior a 0.2. Los resultados de las pruebas realizadas exhiben que el IMP es una prueba confiable y con poder de discriminación satisfactorio. En la tabla 1 se muestran las cinco actividades que predicen mayormente el éxito de los estudiantes frente al SMR asociado a la resolución de las ED de segundo orden.

\section{Tabla 1.}

Las cinco actividades que predicen mayormente el éxito de los estudiantes frente al SMR.

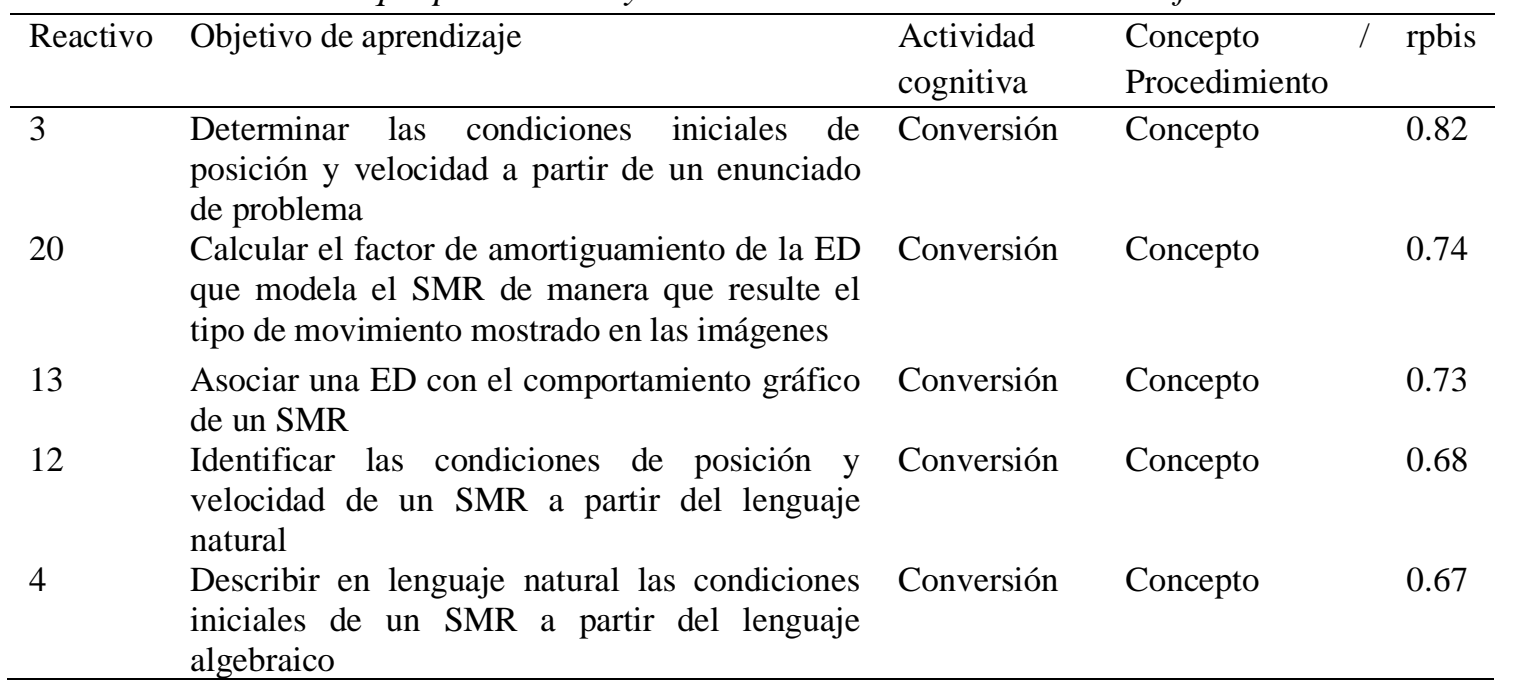


A continuación (figura 2) como ejemplo se muestra el reactivo 13, se trata de un reactivo conceptual y que implica una AC de conversión del lenguaje natural y algebraico al gráfico para su respectiva solución, es medianamente difícil (ID $=0.45$ ), altamente discriminatorio (IDC $=0.73$ ) y predictivo en alto grado (rpbis=0.73). También se observa que en general los reactivos predictivos son medianamente difíciles o de dificultad media y con alta discriminación.

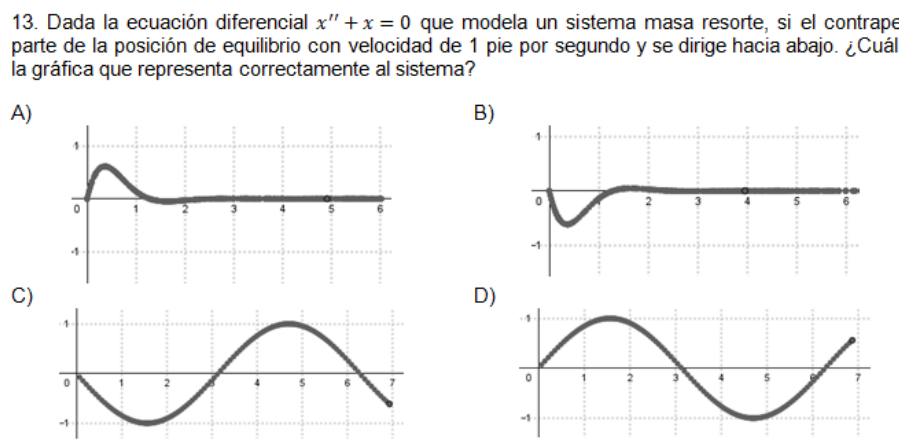

Figura 2. Reactivo 13 del instrumento de medición post-test.

Una prueba $\mathrm{T}$ de muestras independientes entre los reactivos de corte conceptual y los reactivos de tipo procedimental mostró diferencias significativas $(p=0.037)$, los estadísticos de grupo muestran (tabla 2) también que el éxito de los estudiantes radica en trabajar con materiales que incluyan actividades que tengan un balance en las AC y el uso de los diferentes registros de representación, además de hacer énfasis en los aspectos conceptuales del OM.

Tabla 2.

Estadísticas de grupo en relación al coeficiente de correlación biserial

\begin{tabular}{|c|c|c|c|c|}
\hline $\begin{array}{l}\text { Tipo } \\
\text { Reactivo }\end{array}$ & $\begin{array}{l}\text { de } \begin{array}{l}\text { Número } \\
\text { reactivos }\end{array}\end{array}$ & de & $\begin{array}{l}\text { Desviación } \\
\text { Estándar }\end{array}$ & $\begin{array}{l}\text { Error de desviación } \\
\text { promedio }\end{array}$ \\
\hline Conceptual & 11 & .5955 & .1837 & .0554 \\
\hline Procedimental & 7 & .4186 & .1118 & .0422 \\
\hline
\end{tabular}

El programa interactivo diseñado en GeoGebra permitió a los estudiantes visualizar simultáneamente los desplazamientos del contrapeso y asociarlo al comportamiento gráfico, lo que promovió que realizaran algunas asociaciones geométricas importantes que difícilmente se pudieran lograr si no se cuenta con tecnología, aunque también se evidenció la necesidad de reforzar la relación del signo de la pendiente de la recta tangente a la gráfica de tiempo contra posición con la dirección del contrapeso, así como también observar la determinación de posiciones extremas con máximos o mínimos relativos y el algoritmo para su respectiva determinación.

A partir de los resultados del IMP se encontró que la SA favorece en los estudiantes las siguientes habilidades: identificar el tipo de movimiento a partir de una representación gráfica, identificar el tipo de movimiento del SMR a partir de un conjunto de datos numéricos, identificar el desplazamiento extremo a partir de un conjunto de datos numéricos, calcular la frecuencia de un SMR a partir del valor de la masa y el estiramiento del resorte, y describir en lenguaje natural el comportamiento del contrapeso (posición, dirección y velocidad) a partir de una representación gráfica. 
Con GeoGebra, es posible crear aplicaciones interactivas que se pueden usar como herramientas de enseñanza para ilustrar los OM (Caligaris et al., 2015). Estas herramientas virtuales ofrecen grandes campos de oportunidad para su aprovechamiento en las metodologías de enseñanza-aprendizaje, debido a su versatilidad para visualizar los OM con alto grado de abstracción, además ofrecen aportaciones importantes a la ingeniería aplicada (González, Gutiérrez y Sandoval, 2017). En contraste también se determinaron algunas habilidades que deben fortalecerse en los estudiantes, como lo son: asociar una representación gráfica de un SMR con su ED y viceversa, asociar una representación gráfica de un SMR con su ED y las condiciones iniciales de posición y velocidad, determinar las condiciones iniciales de posición y velocidad a partir de un enunciado de problema.

\section{Conclusiones}

En esta investigación se rescata la visualización en el sentido de ilustrar el OM desde sus diferentes representaciones. Una característica peculiar de la SA es que se recurre a representar virtualmente y gráficamente el SMR, en el cual es posible variar la masa, rigidez, amortiguamiento, posición y velocidad inicial. Con tales movimientos se visualizan tanto el efecto de manera virtual como directamente en el comportamiento gráfico asociado, la pendiente de la recta tangente, entre otros atributos del concepto de derivada. En otras palabras, la SA incorpora un dispositivo tecnológico que a través de la interacción dote de sentido a los OM y se enfatice en sus atributos y regularidades.

Como se describe al inicio del documento, este trabajo reporta los resultados de una investigación realizada al poner en escena una SA distinta a la tradicional en el curso de ED. Algunos factores importantes que se encontraron y afectan el proceso de enseñanza-aprendizaje fueron: el aislamiento social ya que no permite la interrelación alumno-docente de manera presencial, deficiencia en la conectividad y los equipos de cómputo, así como los aspectos sociales, psicológicos y de salud.

Se construyó un IMD con el propósito de indagar sobre los antecedentes de los estudiantes sujetos a esta investigación, se determinó su coeficiente de confiabilidad $(r=0.80)$ con el método KR-20, también se calculó la distribución de los puntajes totales mediante la prueba delta de Ferguson (0.96), dichos valores son aceptables de acuerdo a los especialistas. Con base en los resultados de este IMD se encontró que los estudiantes tienen dificultades para traducir al lenguaje natural una ED de primer orden, identificar el concepto de derivada y determinar el tipo de solución que produce una ED de segundo grado.

Con base en la TRS de Duval se diseñó e implementó una SA que incorpora un programa interactivo diseñado con el $\mathrm{SG}$, con el propósito de mejorar los conocimientos y habilidades de los estudiantes en términos de las AC, las distintas representaciones semióticas y los conceptos asociados a las aplicaciones de las ED de segundo orden en el SMR. La SA diseñada enfatiza en el uso de los diferentes registros de representación. Se incluye el SG a la SA para que el estudiante construya los OM a partir de la vinculación de los distintos registros de representación, por lo que la TRS juega un papel primordial a partir de las AC. Para el diseño de la SA y el programa interactivo en el SG se consideró la experiencia de profesores que imparten cursos de ED, los aportes bibliográficos sobre las buenas prácticas con el SG y los resultados del IMD. 
La revisión y análisis de las respuestas de los estudiantes en las hojas de trabajo a partir del uso del programa interactivo permitió observar y admitir logros importantes, como: asociar adecuadamente el signo de la velocidad o pendiente de la recta tangente del contrapeso con su dirección independientemente del tipo de movimiento del SMR, vincular el número de los cortes de la gráfica con respecto al eje horizontal de referencia con el número de veces que el contrapeso pasa por la condición de equilibrio, ligar el comportamiento de la gráfica con la estabilización del sistema y la posición del contrapeso en el eje de referencia, relacionar de manera correcta el movimiento vertical del contrapeso con el comportamiento oscilatorio de la gráfica. También se detectaron deficiencias en los estudiantes que deben superarse y que quedan como reto para los investigadores de este estudio, como por ejemplo: describir adecuadamente la manera en que se calcula el desplazamiento extremo, toda vez que no lo asociaron al punto crítico, se encontró latente la dificultad para transitar del lenguaje natural al lenguaje algebraico, no lograron determinar la ED y sus condiciones iniciales de posición y de velocidad a partir del problema, y se detectaron errores de manipulación algebraica.

Con el propósito de validar el logro de los estudiantes en torno a los objetivos de aprendizaje planteados, sus habilidades en términos de las AC y el uso de distintos registros de representación se diseñó y aplicó un IMP. Se calculó su confiabilidad mediante el método KR$20(r=0.86)$ y también se determinó la distribución de los puntajes totales mediante la prueba delta de Ferguson (0.95), los valores satisfacen ampliamente los sugeridos por los expertos. La validez de contenido tanto del diagnóstico como del IMP se garantizó con la participación de jueces expertos y método de consenso grupal, por lo que son válidos y confiables.

La puesta en escena de la SA asistida con GeoGebra se considera exitosa en varios aspectos, por una parte se logra que los estudiantes se apropien intelectualmente de las aplicaciones de las ED de segundo orden asociadas al SMR y los conceptos matemáticos asociados, su participación con las actividades, hojas de trabajo y los resultados de los instrumentos de medición es muestra de ello.

La utilización de la SA y las aplicaciones en GeoGebra permiten utilizar el tiempo de manera más eficiente, toda vez que la manipulación numérica y algebraica queda en segundo plano, en la SA se privilegia el tránsito entre los diferentes registros de representación y se promueve el OM con una perspectiva más conceptual, además de enfatizar en las habilidades de experimentación, exploración, análisis e interpretación de resultados.

En general la SA propuesta promueve la vinculación de los distintos registros de representación, así como el fortalecimiento de la AC de conversión. De manera puntual se logran objetivos de aprendizaje asociados al SMR y las ED de segundo orden. Se observa que el éxito de los estudiantes se logra mayormente a través del fortalecimiento de los aspectos conceptuales y la AC de conversión de los OM asociados al SMR.

Los análisis realizados también permitieron detectar deficiencias importantes en las transformaciones del lenguaje natural a otros registros de representación, lo cual confirma las dificultades que los estudiantes tienen para modelar a través de las ED. La experiencia de esta investigación y los resultados obtenidos indican la necesidad de fortalecer la enseñanza de las ED a través de SA que promuevan un balance en el uso y vinculación dinámica de los registros de representación, acercamientos intuitivos a los conceptos matemáticos y el uso de recursos 
tecnológicos que permitan al estudiante la exploración, la conjetura y la validación de sus procedimientos y resultados.

\section{Referencias}

Abbey, K. D. (2008). Students' understanding of deriving properties of a function's graph from the sign chart of the first derivative (Doctoral dissertation, University of Maine). Recuperado http://citeseerx.ist.psu.edu/viewdoc/download?doi=10.1.1.611.9646\&rep=rep1\&type=p df

Arango, J., Gaviria, D. \& Valencia, A. (2015). Differential Calculus Teaching through Virtual Learning Objects in the Field of Management Sciences. Procedia - Social and Behavioral Sciences, 176, 412-418. doi: https://doi.org/10.1016/j.sbspro.2015.01.490

Areaya, S. \& Sidelil, A. (2012). Students' difficulties and misconceptions in learning concepts of limit, continuity and derivative. The Ethiopian Journal of Education, 32(2), 1-38. Recuperado de http://ejol.aau.edu.et/index.php/EJE/article/view/215/189

Backhoff, E., Larrazolo, N. \& Rosas, M. (2000). Nivel de dificultad y poder de discriminación del Examen de Habilidades y Conocimientos Básicos (EXHCOBA). Revista Electrónica de Investigación Educativa, 2 (1). Recuperado de https://redie.uabc.mx/redie/article/view/15

Báez, N., Blanco, R. \& Pérez, O. (2015a). Dificultades de los alumnos en el trabajo con los conceptos del cálculo diferencial. Acta Latinoamericana de Matemática Educativa, 28, 57-63. Recuperado de http://funes.uniandes.edu.co/10707/

Báez, N., Blanco, R. \& Pérez, O. (2015b). Fundamentación teórica de la apropiación conceptual con ayuda de las Tics, ejemplificando en la derivada. Acta Latinoamericana de Matemática Educativa, 28, 1576-1582.

Bhagat, K. K. \& Chang, C. (2015). Incorporating GeoGebra into Geometry Learning-A lesson from India. Eurasia Journal of Mathematics, Science and Technology Education, 11(1), 77-86. doi: https://doi.org/10.12973/eurasia.2015.1307a

Caligaris, M., Schivo, M. \& Romiti, M. (2015). Calculus \& GeoGebra, an interesting partnership. Procedia - Social and Behavioral Sciences, 174, 1183-1188. doi: https://doi.org/10.1016/j.sbspro.2015.01.735

Chen, C. L. \& Wu, C. C. (2020). Students' behavioral intention to use and achievements in ICTIntegrated mathematics remedial instruction: Case study of a calculus course. Computers \& Education, $145 . \quad$ doi: https://doi.org/10.1016/j.compedu.2019.103740

Cistac, G., Bongianino, R., Filippi, J. \& Kovac, F. (2009). La simulación como medio de interrelación entre herramientas matemáticas y procesos tecnológicos. Formación Universitaria, 2 (5), 3-11. doi: http://dx.doi.org/10.4067/S0718-50062009000500002

Contreras, L. A. \& Backhoff, E. (2004). Metodología para elaborar exámenes criteriales alineados al currículo, en Castañeda, S. (ed.), Educación aprendizaje y cognición, teoría en la práctica. México: Manual Moderno.

Corral, Y. (2009). Validez y confiabilidad de los instrumentos de investigación para la recolección de datos. Revista Ciencias de la Educación, 19 (33), 228-247. Recuperado de http://servicio.bc.uc.edu.ve/educacion/revista/n33/art12.pdf

Córdoba, F., Castrillón, E. \& Rojas, C. (2015). Geogebra como herramienta de apoyo visual en la solución de problemas de modelación en matemática escolar. Acta Latinoamericana de Matemática Educativa, 28, 1725-1730. Recuperado de http://funes.uniandes.edu.co/11053/1/Cordoba2015Geogebra.pdf 
Crocker, L. \& Algina, J. (1986). Introduction to Classical and Modern Test Theory. Holt, Rinehart and Winston Ed.

Ding, L., Chabay, R., Sherwood, B. \& Beichner R. (2006). Evaluating an electricity and magnetism assessment tool: Brief electricity and magnetism assessment. Physical Review Special Topics - Physics Education Research, 2 (1), 1-7. doi: http://dx.doi.org/10.1103/PhysRevSTPER.2.010105

Duval, R. (2000). Representación, visión y visualización: Funciones cognitivas en el pensamiento matemático. Recuperado de http://www.matedu.cinvestav.mx/elibrosydoc/pme-procee.pdf

Duval, R. (2006a). A cognitive analysis of problems of comprehension in a learning of mathematics. Journal of Educational Studies in Mathematic, 61 (1-2), 103-131. doi: http://dx.doi.org/10.1007/s10649-006-0400-z

Duval, R. (2006b). Quelle Sémiotique Pour L'Analyse de L'Activité et des productions mathématiques. Revista Latinoamericana de Investigación en Matemática Educativa, 9, 45-81. Recuperado de https://dialnet.unirioja.es/servlet/articulo?codigo $=2161528$

Engelhardt, P. (2009). An Introduction to Classical Test Theory as Applied to Conceptual Multiple-Choice Tests in Getting Started in PER, 2. Recuperado de http://www.compadre.org/Repository/document/ServeFile.cfm?ID=8807\&DocID=1148

Fatimah, R., Norazzila, S. \& Rohani, A. (2013). Exploring Student's in-depth Learning Difficulties in Mathematics through Teachers' Perspective. Procedia - Social and Behavioral Sciences, 97, 339-345. doi: http://dx.doi.org/10.1016/j.sbspro.2013.10.243

Feo, R. (2010). Orientaciones básicas para el diseño de estrategias didácticas. Tendencias pedagógicas, 26, 220-236. Recuperado de https://www.researchgate.net/publication/48523396_Orientaciones_basicas_para_el_dis eno_de_estrategias_didacticas

Gómez-Blancarte, A., Guirette, R. \& Morales-Colorado, F. (2017). Propuesta para el tratamiento de interpretación global de la función cuadrática mediante el uso del software Geogebra. Educación Matemática, 29 (3), 189-224. doi: https://doi.org/10.24844/EM2903.07

González, J. V., Gutiérrez, R. D. \& Sandoval, M. (2017). Desarrollo didáctico con GeoGebra como herramienta para la enseñanza en aplicaciones de mecanismos y diseño de maquinaria dentro de la ingeniería. XXIII congreso internacional anual de la SOMIM. Recuperado de http://revistasomim.net/congreso2017/articulos/A5_175.pdf

Guerrero-Ortiz, C., Mejía-Velasco, H. R., \& Camacho-Machín, M. (2016). Representations of a mathematical model as a means of analyzing growth phenomena. The Journal of Mathematical Behavior, 42, 109-126. doi: https://doi.org/10.1016/j.jmathb.2016.03.001

Habre, S. \& Abboud, M. (2006). Student's conceptual understanding of a function and its derivative in an experimental calculus course. Journal of Mathematical Behavior, 25 (1), 57 72. doi: https://doi.org/10.1016/j.jmathb.2005.11.004

Hamidreza, K., Zaleha, I. \& Yudariah, M. (2012). Engineering Mathematics Obstacles and Improvement: A comparative study of students and lecturers perspectives through creative problem solving. Procedia - Social and Behavioral Sciences, 56, 556-564. doi: https://doi.org/10.1016/j.sbspro.2012.09.688

Hashemi, N., Abu, M. S., Kashefi, H. \& Rahimi, K. (2014). Undergraduate Students' Difficulties in Conceptual Understanding of Derivation. Procedia - Social and Behavioral Sciences, 143, 358-366. doi: https://doi.org/10.1016/j.sbspro.2014.07.495

Henrysson, S. (1971). Gathering, Analysing, and Using Data on Teste Items. En R.L. Thorndike (Ed.), Educational Measurement. Washington, DC: American Council on Education. 
Molina, A. I., Wizner, Á. L., Lacave, C. \& Gallardo, J. (2015). Una Herramienta de diseño y análisis de instrumentos de evaluación e indagación docente. A: JENUI 2015. Actas de las XXI Jornadas de la Enseñanza Universitaria de la Informática. Universitat Oberta La Salle ed. Andorra la Vella: Universitat Oberta La Salle, 2015, 144-151. Recuperado de http://hdl.handle.net/2117/76924

Nazihatulhasanah, A. \& Nurbiha, S. (2015). The effects of GeoGebra on students achievement. Procedia - Social and Behavioral Sciences, 172, 208-214. doi: https://doi.org/10.1016/j.sbspro.2015.01.356

PISA. (2012). Resultados de PISA 2012, Grupo Fórmula. Recuperado de https://www.oecd.org/pisa/keyfindings/PISA-2012-results-mexico-ESP.pdf

PISA. (2015). Resultados de PISA 2015, Grupo Fórmula. Recuperado de https://www.oecd.org/pisa/pisa-2015-results-in-focus-ESP.pdf

Ponce, J. C., Matthews, K. E. \& Adams, P. (2018). On the use of history of mathematics: an introduction to Galileo's study of free fall motion. International Journal of Mathematical Education in Science and Technology, 49 (4), 517-529. doi: https://doi.org/10.1080/0020739X.2017.1377301

Saha, R. A., Ayub, A. F. M. \& Tarmizi, R. A. (2010). The effects of GeoGebra on mathematics achievement: enlightening coordinate geometry learning. Procedia-Social and Behavioral Sciences, 8, 686-693. doi: https://doi.org/10.1016/j.sbspro.2010.12.095

Sfard, A. (1991). On the dual nature of mathematical conceptions: reflections on processes and objects as different sides of the same coin. Educational Studies in Mathematics, 22, 136. doi: https://doi.org/10.1007/BF00302715

Torres, C. A. \& Racedo, D. M. (2014). Estrategia didáctica mediada por el software GeoGebra para fortalecer la enseñanza-aprendizaje de la geometría en estudiantes de $9^{\circ}$ de básica secundaria. Magister en educación. Universidad de la Costa, Barranquilla, Colombia. Recuperado de https://repositorio.cuc.edu.co/handle/11323/1284

Zengin, Y., Furkan, H. \& Kutluca, T. (2012). The effect of dynamic mathematics software GeoGebra on student achievement in teaching of trigonometry. Procedia - Social and Behavioral Sciences, 31, 183-187. doi: https://doi.org/10.1016/j.sbspro.2011.12.038

Zerrin, R. \& Sebnem, O. (2010). Using GeoGebra as an information technology tool: parabola teaching. Procedia - Social and Behavioral Sciences, 9, 565-572. doi: https://doi.org/10.1016/j.sbspro.2010.12.198. 